\title{
ANÁLISE DO MERCADO DE PROCEDIMENTOS ESTÉTICOS: A IMPORTÂNCIA DE CONHECER SEU PÚBLICO-ALVO
}

\author{
Amanda Aparecida de Paula, Gustavo Yuho Endo, Marco Antonio Catussi Paschoalotto \\ Universidade do Oeste Paulista - UNOESTE, Curso de Administração, Presidente Prudente, SP. E-mail: \\ amanda_depaula@hotmail.com
}

\section{RESUMO}

A busca pelo bem-estar torna o mercado de estética altamente promissor e aumenta o interesse de investidores no segmento. A análise de mercado, uma das etapas do plano de negócio, permite ao empreendedor conhecer o ambiente que pretende se inserir. Portanto, o objetivo deste trabalho é demonstrar a importância de realizar a análise de mercado para compreender o mercado de estética e conhecer o público-alvo de Presidente Prudente. Como método, foi realizada uma pesquisa quantitativa, por meio de uma survey por amostragem não probabilística e utilização da técnica de estatística descritiva para análise dos dados. Os resultados demonstram que a qualidade dos serviços é exigência entre os consumidores, também que o público masculino tem relevante potencial de consumo e a necessidade de buscar diferencial no mercado. Por fim, constatou que os resultados da análise de mercado são fundamentais na abertura de um negócio na área.

Palavras-chave: Ambiente. Análise de Mercado. Mercado de Estética. Plano de negócio. Públicoalvo.

\section{MARKET ANALYSIS OF AESTHETIC PROCEDURES: THE IMPORTANCE OF KNOWING YOUR TARGET AUDIENCE}

\begin{abstract}
The search for well-being makes the aesthetic market highly promising and increases the interest of investors in the segment. The market analysis, one of the stages of the business plan, allows the entrepreneur to know the ambience that intends to insert himself. Thus, the objective of this work is to demonstrate the importance of using the market analysis to understand the aesthetics market and to know the target audience of Presidente Prudente. As method, a quantitative survey was carried out by means of a non - probabilistic sample survey and using of a descriptive statistical technique data analysis. The results demonstrate that the quality of services is a requirement among consumers, also that the male audience has relevant consumption potential and the need to seek a differential in the market. Finally, he found that the results of the market analysis are fundamental in opening a business in the area.
\end{abstract}

Keywords: Ambience. Market Analysis. Aesthetic Market. Business Plan. Target Audience. 


\section{INTRODUÇÃO}

Para se iniciar um negócio de sucesso não bastam ideias visionárias e persistência, uma vez que, por consequência do processo de globalização o mercado se mantem em constante mudança. Portanto, para sobreviver a essas mudanças é fundamental que o empreendedor faça um planejamento antes de iniciar o seu negócio. Para isso existe o plano de negócio que permite ao empreendedor descrever, por meio de um documento, o percurso a ser percorrido para alcançar seus objetivos, bem como identificar e corrigir possíveis erros antes de entrar no mercado (SEBRAE, 2013).

Um dos propósitos do plano de negócio é conhecer o ambiente onde pretende se inserir. A análise do mercado é uma das etapas para o planejamento estratégico do negócio que possibilita ao empreendedor conhecer e avaliar o ambiente externo e as inter-relações (BIAGIO; BATOCCHIO, 2012). Por meio das informações obtidas na análise de mercado é possível traçar o perfil dos potenciais clientes, desenvolver estratégias, observar as características dos concorrentes, identificar ameaças e oportunidades, o que torna o processo decisório mais preciso (DESMEDT, et. al., 2016).

A busca pelo bem-estar fez com que o consumo de produtos e serviços do mercado de estética crescesse consideravelmente em todas as classes sociais, o que torna este setor um dos que mais tem crescido no país (SEBRAE, 2017), e que, por consequência, reflete no número de empreendedores dispostos a investir nesse mercado altamente promissor. Para tanto, mais do que possuir habilidades técnicas, é essencial que o empreendedor saiba administrar profissionalmente o seu negócio.

O objetivo desse artigo é demonstrar a importância de realizar a análise de mercado para compreender o mercado de estética e conhecer o público-alvo de Presidente Prudente.

\section{METODOLOGIA}

Quanto à classificação da pesquisa, trata-se do método quantitativo. Este tipo de pesquisa visa, por meio de uma análise estatística, quantificar dados coletados de grandes amostras (RODRIGUES, 2015). A classificação desse estudo é não-probabilística, pois a amostra oferece características da população, porém os resultados não podem ser generalizados (MALHOTRA, 2011).

O procedimento utilizado para coleta de dados foi a pesquisa survey. A survey é usada para estudar a parcela de uma população por meio de entrevistas ou questionários (BABBIE, 2003). Nesse estudo foi utilizado o questionário com perguntas fechadas.

Foi utilizada a técnica de estatística descritiva para a análise dos dados. Segundo Castanheira (2016), "a estatística descritiva nos permite fazer um estudo completo e detalhado sobre determinada população ou amostra dela".

Para a criação do questionário foi utilizado o Google Forms. Em seguida, o link do questionário foi disponibilizado nas redes sociais, que ficou disponível no período de 16 de abril a 7 de maio de 2018 e, no final do período, totalizou 309 respondentes de Presidente Prudente, o público-alvo dessa pesquisa.

\section{RESULTADOS}

A amostra do estudo foi composta por 309 indivíduos, sendo 82,2\% predominantemente representado pelo público feminino e $17,8 \%$ do sexo masculino. Conforme a Tabela 1, em relação a faixa de idade, foi possível verificar que os indivíduos que responderam ao questionário, em maioria, possuem de 19 a 29 anos em ambos os sexos. 
Tabela 1. Distribuição de sexo e idade dos participantes da pesquisa.

\begin{tabular}{c|c|c|c|c|c|c|c}
\hline Sexo/Idade & $\begin{array}{c}\text { Até } 18 \\
\text { anos }\end{array}$ & $\begin{array}{c}\text { De } 19 \text { a } \\
29 \text { anos }\end{array}$ & $\begin{array}{c}\text { De } 30 \text { a } 39 \\
\text { anos }\end{array}$ & $\begin{array}{c}\text { De } 40 \text { a } \\
49 \text { anos }\end{array}$ & $\begin{array}{c}\text { De } 50 \text { a } \\
59 \text { anos }\end{array}$ & $\begin{array}{c}60 \text { anos } \\
\text { ou mais }\end{array}$ & Total \\
\hline Feminino & $7,4 \%$ & $45,6 \%$ & $13,9 \%$ & $8,7 \%$ & $5,8 \%$ & $0,6 \%$ & $82,2 \%$ \\
\hline Masculino & $1,0 \%$ & $10,7 \%$ & $2,6 \%$ & $2,6 \%$ & $1,0 \%$ & $0,0 \%$ & $17,8 \%$ \\
\hline Total & $8,4 \%$ & $56,3 \%$ & $16,5 \%$ & $11,3 \%$ & $6,8 \%$ & $0,6 \%$ & $100,0 \%$ \\
\hline
\end{tabular}

Fonte: Elaborado pelos autores (2018)

Já a Tabela 2 apresenta a distribuição de sexo e renda. 0 maior percentual de ambos os sexos é na faixa de $\mathrm{R} \$ 955,00$ a $\mathrm{R} \$ 1.909,00$, que representa $29,8 \%$ do total de participantes.

Tabela 2. Distribuição de sexo e renda dos participantes da pesquisa.

\begin{tabular}{c|c|c|c|c|c|c|c|c}
\hline Sexo/Renda & $\begin{array}{c}\text { Até } \\
\mathrm{R} \$ \\
954\end{array}$ & $\begin{array}{c}\mathrm{R} \$ 955 \\
\mathrm{a} \mathrm{R} \$ \\
1.909\end{array}$ & $\begin{array}{c}\mathrm{R} \$ 1.910 \\
\mathrm{a} \mathrm{R} \$\end{array}$ & $\begin{array}{c}\mathrm{R} \$ 2.865 \\
\mathrm{a} \mathrm{R} \$\end{array}$ & $\begin{array}{c}\mathrm{R} \$ 3.819 \\
\mathrm{a} \mathrm{R} \$ \\
4.864\end{array}$ & $\begin{array}{c}\mathrm{R} \$ 4.774 \\
\text { a } \mathrm{R} \$ \\
5.728\end{array}$ & $\begin{array}{c}\text { Acima } \\
\text { de } \mathrm{R} \$ \\
5.728\end{array}$ & Total \\
\hline Feminino & $22,0 \%$ & $24,3 \%$ & $12,9 \%$ & $7,1 \%$ & $3,6 \%$ & $6,1 \%$ & $6,1 \%$ & $82,2 \%$ \\
\hline Masculino & $5,2 \%$ & $5,5 \%$ & $2,9 \%$ & $1,3 \%$ & $0,3 \%$ & $0,6 \%$ & $1,9 \%$ & $17,8 \%$ \\
\hline Total & $27,2 \%$ & $29,8 \%$ & $15,9 \%$ & $8,4 \%$ & $3,9 \%$ & $6,8 \%$ & $8,1 \%$ & $100 \%$ \\
\hline
\end{tabular}

Fonte: Elaborado pelos autores (2018)

A Tabela 3 mostra que os participantes da pesquisa do sexo masculino não estão dispostos a pagar mais do que $\mathrm{R} \$ 250,00$ com procedimentos estéticos por mês, já do sexo feminino $2,9 \%$ estão dispostos a gastar acima de $\mathrm{R} \$ 300,00$. Ambos concordam em uma média de gasto de $\mathrm{R} \$ 51,00$ a $\mathrm{R} \$ 100,00$ (mensal), representando 40,5\% em comparação aos demais valores.

Tabela 3. Distribuição de sexo o quanto estão dispostos a gastar com procedimento estéticos por mês.

\begin{tabular}{|c|c|c|c|c|c|c|c|c|}
\hline Sexo/Gastar & $\begin{array}{c}\text { Até } \\
\mathrm{R} \$ 50\end{array}$ & $\begin{array}{l}\mathrm{R} \$ 51 \mathrm{a} \\
\mathrm{R} \$ 100\end{array}$ & $\begin{array}{c}\mathrm{R} \$ 101 \mathrm{a} \\
\mathrm{R} \$ 150\end{array}$ & $\begin{array}{c}\mathrm{R} \$ 151 \mathrm{a} \\
\mathrm{R} \$ 200\end{array}$ & $\begin{array}{c}\mathrm{R} \$ 201 \mathrm{a} \\
\mathrm{R} \$ 250\end{array}$ & $\begin{array}{c}\mathrm{R} \$ 251 \mathrm{a} \\
\mathrm{R} \$ 300\end{array}$ & $\begin{array}{c}\text { Acima } \\
\text { de } R \$ \\
300\end{array}$ & Total \\
\hline Feminino & $10,4 \%$ & $31,7 \%$ & $15,5 \%$ & $10,7 \%$ & $4,5 \%$ & $6,5 \%$ & $2,9 \%$ & $82,2 \%$ \\
\hline Masculino & $3,2 \%$ & $8,7 \%$ & $4,5 \%$ & $0,6 \%$ & $0,6 \%$ & - & - & $17,8 \%$ \\
\hline Total & $13,6 \%$ & $40,5 \%$ & $20,1 \%$ & $11,3 \%$ & $5,2 \%$ & $6,5 \%$ & $2,9 \%$ & $100 \%$ \\
\hline
\end{tabular}

Fonte: Elaborado pelos autores (2018)

De acordo com a Tabela 4, a maioria dos participantes da pesquisa é solteiro (35\%) e casado (34,6\%). Entretanto, o estado civil "namorando" é o que mais está disposto a gastar com procedimentos estéticos por mês. 
Tabela 4. Distribuição do estado civil dos participantes da pesquisa e quanto estão dispostos a gastar com procedimentos estéticos por mês.

\begin{tabular}{c|c|c|c|c|c|c|c|c}
\hline $\begin{array}{c}\text { Gastar / } \\
\text { Estado Civil }\end{array}$ & $\begin{array}{c}\text { Até } \\
\mathrm{R} \$ 50\end{array}$ & $\begin{array}{c}\mathrm{R} \$ 51 \mathrm{a} \\
\mathrm{R} \$ 100\end{array}$ & $\begin{array}{c}\mathrm{R} \$ 101 \mathrm{a} \\
\mathrm{R} \$ 150\end{array}$ & $\begin{array}{c}\mathrm{R} \$ 151 \mathrm{a} \\
\mathrm{R} \$ 200\end{array}$ & $\begin{array}{c}\mathrm{R} \$ 201 \mathrm{a} \\
\mathrm{R} \$ 250\end{array}$ & $\begin{array}{c}\mathrm{R} \$ 251 \mathrm{a} \\
\mathrm{R} \$ 300\end{array}$ & $\begin{array}{c}\text { Acima } \\
\text { de } \mathrm{R} \$ \\
300\end{array}$ & Total \\
\hline Solteiro(a) & $5,5 \%$ & $14,2 \%$ & $8,1 \%$ & $2,6 \%$ & $1,6 \%$ & $2,3 \%$ & $0,6 \%$ & $35 \%$ \\
\hline Namorando & $2,9 \%$ & $12,6 \%$ & $4,2 \%$ & $1,3 \%$ & $1,0 \%$ & $0,6 \%$ & $1,0 \%$ & $23,6 \%$ \\
\hline Noivo(a) & $0,3 \%$ & $1,0 \%$ & $1,6 \%$ & $0,6 \%$ & $0,3 \%$ & $0,3 \%$ & - & $4,2 \%$ \\
\hline Casado(a) & $4,9 \%$ & $11,7 \%$ & $5,8 \%$ & $6,5 \%$ & $1,9 \%$ & $3,2 \%$ & $0,6 \%$ & $34,6 \%$ \\
\hline Divorciado & - & $0,6 \%$ & - & $0,3 \%$ & $0,3 \%$ & - & $0,6 \%$ & $1,9 \%$ \\
\hline Viúvo(a) & - & $0,3 \%$ & $0,3 \%$ & - & - & - & - & $0,6 \%$ \\
\hline Total & $13,6 \%$ & $40,5 \%$ & $20,1 \%$ & $11,3 \%$ & $5,2 \%$ & $6,5 \%$ & $2,9 \%$ & $100 \%$ \\
\hline
\end{tabular}

Fonte: Elaborado pelos autores (2018)

Conforme demonstra a Tabela 5, os participantes com a distribuição de renda inferior a $\mathrm{R} \$ 3.818,00$ estão mais dispostos a gastar com procedimentos estéticos acima de $\mathrm{R} \$ 300,00$ por mês em comparação aos respondentes que tem renda acima de 5.728,00.

Tabela 5. Distribuição da renda mensal dos participantes da pesquisa e quanto estão dispostos a gastar com procedimentos estéticos por mês.

\begin{tabular}{|c|c|c|c|c|c|c|c|c|}
\hline $\begin{array}{c}\text { Gastar / } \\
\text { Renda }\end{array}$ & $\begin{array}{c}\text { Até } \\
\mathrm{R} \$ 50\end{array}$ & $\begin{array}{l}R \$ 51 a \\
R \$ 100\end{array}$ & $\begin{array}{c}\mathrm{R} \$ 101 \mathrm{a} \\
\mathrm{R} \$ 150\end{array}$ & $\begin{array}{c}\mathrm{R} \$ 151 \mathrm{a} \\
\mathrm{R} \$ 200\end{array}$ & $\begin{array}{c}\mathrm{R} \$ 201 \mathrm{a} \\
\mathrm{R} \$ 250\end{array}$ & $\begin{array}{c}\mathrm{R} \$ 251 \mathrm{a} \\
\mathrm{R} \$ 300\end{array}$ & $\begin{array}{c}\text { Acima de } \\
R \$ 300\end{array}$ & Total \\
\hline Até $\mathrm{R} \$ 954$ & $6,1 \%$ & $14,9 \%$ & $3,9 \%$ & $0,3 \%$ & $0,6 \%$ & $0,6 \%$ & $0,6 \%$ & $27,2 \%$ \\
\hline $\begin{array}{l}\mathrm{R} \$ 955 \mathrm{a} \\
\mathrm{R} \$ 1.909\end{array}$ & $4,2 \%$ & $12,0 \%$ & $7,4 \%$ & $3,9 \%$ & $0,6 \%$ & $1,0 \%$ & $0,6 \%$ & $29,8 \%$ \\
\hline $\begin{array}{c}\mathrm{R} \$ 1.910 \mathrm{a} \\
\mathrm{R} \$ 2.864\end{array}$ & $1,0 \%$ & $4,9 \%$ & $4,5 \%$ & $2,6 \%$ & $0,6 \%$ & $1,9 \%$ & $0,3 \%$ & $15,9 \%$ \\
\hline $\begin{array}{c}R \$ 2.865 a \\
R \$ 3.818\end{array}$ & $0,6 \%$ & $3,9 \%$ & $1,0 \%$ & $1,0 \%$ & 0,6 & $1,0 \%$ & $0,3 \%$ & $8,4 \%$ \\
\hline $\begin{array}{c}R \$ 3.819 a \\
R \$ 4.773\end{array}$ & $0,3 \%$ & $1,3 \%$ & $1,0 \%$ & $0,3 \%$ & $0,6 \%$ & $0,3 \%$ & - & $3,9 \%$ \\
\hline $\begin{array}{c}\mathrm{R} \$ 4.774 \mathrm{a} \\
\mathrm{R} \$ 5.728\end{array}$ & $1,0 \%$ & $1,3 \%$ & $1,3 \%$ & $1,0 \%$ & $1,6 \%$ & $0,6 \%$ & - & $6,8 \%$ \\
\hline $\begin{array}{l}\text { Acima de } \\
R \$ 5.728\end{array}$ & $0,3 \%$ & $2,3 \%$ & $1,0 \%$ & $2,3 \%$ & $0,3 \%$ & $1,0 \%$ & $1,0 \%$ & $8,1 \%$ \\
\hline Total & $13,6 \%$ & $40,5 \%$ & $20,1 \%$ & $11,3 \%$ & $5,2 \%$ & $6,5 \%$ & $2,9 \%$ & $100 \%$ \\
\hline
\end{tabular}

Fonte: Elaborado pelos autores (2018)

Conforme apresentado na Figura 1, dentre os respondentes, $28 \%$ optam pela qualidade dos serviços ao buscar por locais que realizam procedimentos estéticos. Outra variável que faz diferença entre as opções de escolha é o profissional com formação na área (19\%), enquanto que, $3 \%$ é influenciada por meio das redes sociais. 
Figura 1. Critérios para escolha do local para realização de procedimentos estéticos.

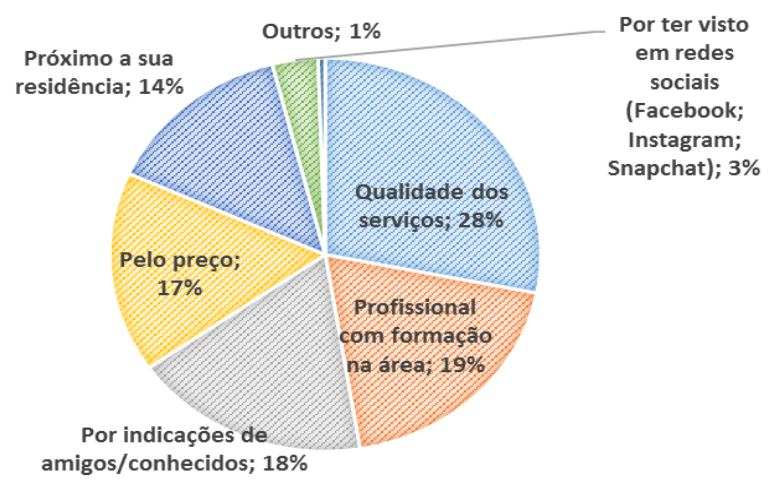

Fonte: Elaborado pelos autores (2018)

De acordo com a Figura 2, a pesquisa apontou que, $50 \%$ dos respondentes tem preferência para realização dos procedimentos estéticos às sexta-feira e aos sábados. Observa-se também que $38 \%$ dos respondentes preferem realizar os procedimentos estéticos no período da tarde (14h01 às 18h00).

Figura 2. O dia da semana preferível para realizar os procedimentos estéticos.
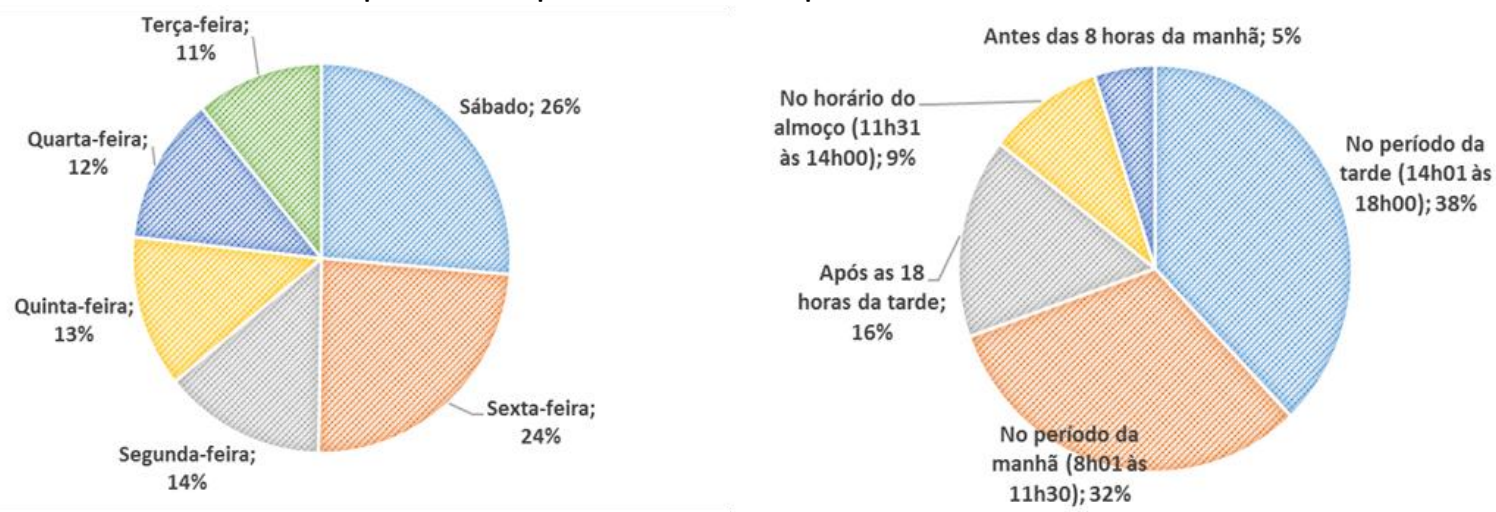

Fonte: Elaborado pelos autores (2018)

Conforme Figura 3, em relação ao atendimento, 83\% dos respondentes preferem ir até o estabelecimento para a realização dos procedimentos estéticos, e desses, $97 \%$ tem a preferência que seja com hora marcada. Nota-se ainda que, $17 \%$ dos respondentes possui interesse em atendimento a domicilio.

Figura 3. Em relação ao atendimento.
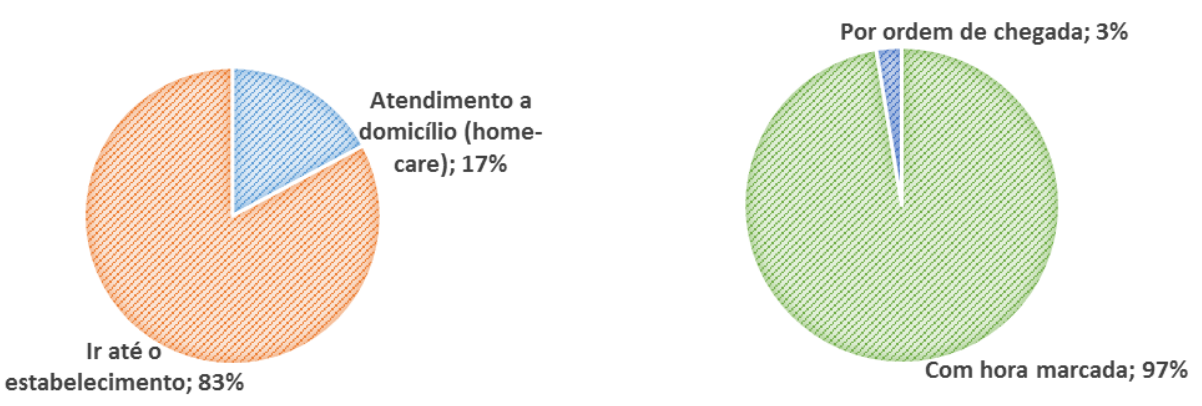

Fonte: Elaborado pelos autores (2018) 
Já a Figura 4, demonstra que $83 \%$ dos respondentes da pesquisa estão "satisfeito" ou "muito satisfeito" com o local que já realizam seus procedimentos estéticos.

Figura 4. Nível de satisfação com o local que já realiza procedimentos estéticos

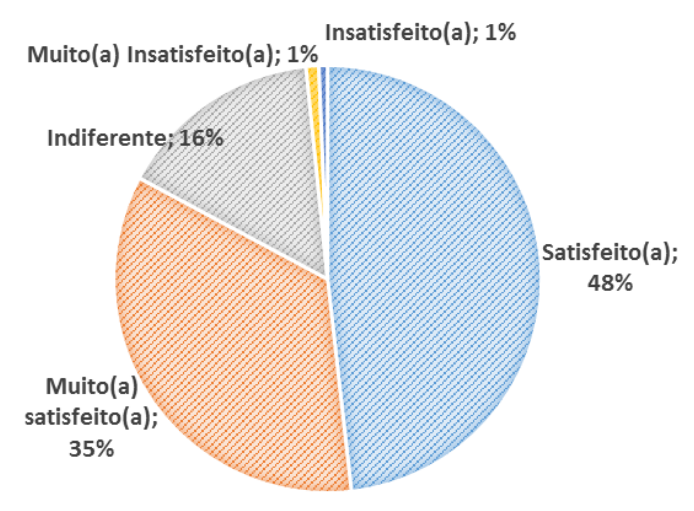

Fonte: Elaborado pelos autores (2018)

A partir dos achados na pesquisa, foram apresentadas diversas informações para a realização da análise de mercado dos procedimentos estéticos de Presidente Prudente/SP. Na próxima seção são apresentadas discussões acerca dos resultados obtidos.

\section{DISCUSSÃO}

$\mathrm{Na}$ amostra dos respondentes de Presidente Prudente/SP, 17,8\% é representada pelo sexo masculino, disposta a pagar até $\mathrm{R} \$ 250,00$ em procedimentos estéticos por mês. O homem, hoje mais vaidoso, demonstra grande potencial de consumo e crescimento no segmento de beleza e estética (SEBRAE, 2015) e é, portanto, uma fatia relevante do mercado a ser explorada por empreendedores dispostos a investir no setor.

Foi possível analisar que, mesmo a rede social sendo uma ferramenta de marketing tão utilizada para atrair clientes, não é critério de destaque para escolher o local para realizar procedimentos estéticos. A influência da escolha está na qualidade dos serviços, diretamente influenciada pela competência de seus profissionais. A importância da qualificação nesse segmento foi determinante para vigorar a Lei no 13.643/18, sancionada em abril de 2018, que exige formação superior ou técnica para o exercício da profissão relacionado à estética (BRASIL, 2018).

Foi observado que $83 \%$ da amostra considera-se "muito satisfeito" ou "satisfeito" com o local onde realizam os procedimentos estéticos, sendo uma provável dificuldade a ser enfrentada por novos entrantes no mercado de estética (DESMEDT, et. al., 2016). Contudo, uma vez identificada as forças da concorrência, é possível traçar estratégias para se diferenciar dos concorrentes (PORTER, 1986). Foi identificado na pesquisa que diferenciais podem ser o cumprimento do horário marcado e também o atendimento da fatia de mercado que se interessa pelo home-care.

\section{CONCLUSÃO}

A proposta da pesquisa foi demonstrar a importância de realizar a análise de mercado para compreender o mercado de estética e conhecer o público-alvo de Presidente Prudente. Por meio da pesquisa de mercado foram obtidos resultados relacionados ao poder de compra, nível de satisfação e características de potenciais clientes dispostos a realizar serviços estéticos. Constatou que estes resultados são fundamentais na abertura de um negócio na área, visto que norteiam as tomadas de decisões e estratégias para se diferenciar dos concorrentes. 
O estudo apresentou limitação quanto à amostragem, visto que, em decorrência da amostra não probabilística não ser representativa, os resultados não podem ser generalizados.

Como sugestão para estudos futuros, propõe-se que sejam feitas pesquisas com amostragem probabilística para que a amostra possa ser representativa. Sugere-se também, expandir a pesquisa para cidades da região.

\section{REFERÊNCIAS}

BABBIE, E. Métodos de pesquisa de survey. 2. ed. Minas Gerais: UFMG, 2003. Disponível em: <https://pt.scribd.com/document/61760031/BABBIE-Earl-Metodos-de-Pesquisa-de-Survey>. Acesso em: 16 jul. 2018.

BIAGIO, L.; BATOCCHIO, A. Plano de negócio: estratégia para micro e pequenas empresas. 2. ed. Barueri, SP: Manole, 2012.

BRASIL. Decreto no 13.643/18, de 4 de abril de 2018. Sancionada lei que regulamenta as profissões de esteticista e de técnico em estética. Câmara dos Deputados, Brasília, DF, 4 abr. 2018. Disponível em: <http://www2.camara.leg.br/camaranoticias/noticias/TRABALHO-EPREVIDENCIA/555478-SANCIONADA-LEI-QUE-REGULAMENTA-AS-PROFISSOES-DE-ESTETICISTA-EDE-TECNICO-EM-ESTETICA.html> Acesso em: 21 jul. 2018.

CASTANHEIRA, N. Cálculo aplicado à gestão e aos negócios. Curitiba: Intersaberes, 2016.

DESMEDT, B, et. al. Overview of skin whitening agents with an insight into the illegal cosmetic market in Europe. Journal of the European Academy of Dermatology and Venerology. V.30, 6, p. 943-950, 2016. https://doi.org/10.1111/jdv.13595

MALHOTRA, N. Pesquisa de marketing: foco na decisão. 3. ed. São Paulo: Pearson Prentice Hall, 2011.

PORTER, M. Estratégia competitiva: técnicas para análise de indústrias e da concorrência. 7. ed. Rio de Janeiro: Elsevier, 1986.

RODRIGUES, A. Pesquisa mercadológica. São Paulo: Pearson Education do Brasil, 2015. SERVIÇO BRASILEIRO DE APOIO ÀS PEQUENAS E MICRO EMPRESAS. A profissionalização dos centros de estética. Brasília, 2017. Disponível em:

<http://www.sebrae.com.br/sites/PortalSebrae/artigos/a-profissionalizacao-dos-centros-deestetica,00a9d62b2b886410VgnVCM1000003b74010aRCRD>. Acesso em: 10 jul. 2018.

SERVIÇO BRASILEIRO DE APOIO ÀS PEQUENAS E MICRO EMPRESAS. Como elaborar um plano de negócios. Brasília, 2013. Disponível em:

<http://www.bibliotecas.sebrae.com.br/chronus/ARQUIVOS_CHRONUS/bds/bds.nsf/5f6dba19ba af17a98b4763d4327bfb6c/\$File/2021.pdf>. Acesso em: 10 jul. 2018 
SERVIÇO BRASILEIRO DE APOIO ÀS PEQUENAS E MICRO EMPRESAS. Boletim tendência e oportunidades mercado de beleza. Brasília, 2015. Disponível em:

<http://www.bibliotecas.sebrae.com.br/chronus/ARQUIVOS_CHRONUS/bds/bds.nsf/9757c668a2 32b1db04e94fb4e56eddba/\$File/5421.pdf>. Acesso em: 20 jul. 2018. 